\title{
How Asthma Becomes a Part of Self: IIIness and Treatment Narratives Based on Patients with Chronic Diseases
}

\author{
Qu Ying ${ }^{1, *}$, Zhang Yidan ${ }^{2}$ \\ ${ }^{1}$ Anthropology, School of Social Development and Public Policy, Fudan University, Shanghai, China \\ ${ }^{2}$ Department of social work, School of Social Development and Public Policy, Fudan University, Shanghai, China
}

\begin{abstract}
Increasing people turn the sufferings of illness into human "errors" and resort to medical science and technology for remedy instead of making meaningful moral responses to illness. This is not completely effective, and it will let them fall into the dilemma of humanitarian spirit. It is necessary to pay attention to the patient's subjective world from the perspective of illness narratives. Based on the process of patient's disease view, the writers explored the mutual influence of disease and the subject. In light with the life course of a patient with chronic disease and the daily experience, the writers started from three aspects: the social environment of disease, the social and cultural significance of disease pain and the process of interaction between patients and asthma, in order to reveal the social and cultural characteristics of chronic disease and how the disease becomes a part of their lives. Disease is interpreted as a kind of narrative reconstruction. By connecting different aspects of life, patients can repair the fracture among their bodies, themselves and the world. It is a kind of subjective treatment that patients give meaning to their personal experience in their own way, and then understand themselves and construct new identities and life scenes.
\end{abstract}

\section{Introduction}

The growing power of medical profession in knowledge, system and social governance deepens the social medicalization, which is manifested in the fact that individual cognition changes from potential patients to real patients and enters the medical system, or some social behaviors and phenomena are defined as illnesses by medicine [1][2]. Medical treatment is no longer limited to clinics, hospitals and other professional medical institutions, but it is widely penetrated into people's daily life [3]. Furthermore, people's motivation in accepting treatment is sometimes not because of the emergency of health hazards, but the urgent desire to improve their lifestyle. Such desire of this particular lifestyle is usually turned into a health problem that can be solved through "medical means", that is, the medicalization of lifestyle [4]. This tendency is equal to the mediacalization of physical problems we encounter in our life. These people regard the medical technology of health care experts and their representatives as a kind of cultural authority, believing that they can help us tide over the difficulties and give us the answers to solve the problems and dilemmas. However, as we all know, medical technology or science and technology cannot help us deal with all the problems and difficulties. Biomedicine and other assistive technologies cannot explain the concept of illness "teleology", let alone the social and humanistic aspects closely related to illness, such as frustration and life pressure. On the contrary, more and more people view the sufferings of illness as the "errors" of human body, and resort to medical science and technology for treatment instead of making meaningful moral (or spiritual) response to the problems of illness. But it deserves our reflection.

In the book by Arthur Kleinman, the Illness Narratives, he distinguishes disease from illness semantically at the very beginning [5]. They are two different worlds. One is the world observed and recorded, and the other is the world experienced and narrated; one is the objective world to find the cause and pathological index, and the other is the subjective world which tells the experience of psychological and social pain. The latter one shows different perspectives in illness narration from the biomedical perspective. It not only regards chronic diseases as a pathological process - the temporary or permanent disorder of patients'biological or social functions. This perspective also focuses on the process of chronic disease depriving patients of the control of their body and the judgment of their life. In addition, patients, family members and wider social relations are living in a vivid experience and are troubled by diseases. This can be a dialogue with the abovementioned dilemma about the lack of humanitarianism in medical solutions, and it is also a case of this paper to present a narrative of chronic diseases.

In addition, as everyone's experience of illness is unique and related to living habits and social environment, the narrative of individual case is meaningful. The body and its internal characteristics are pluralistic and changeable. From the understanding of local biologies, we can know that the intrinsic nature of

\footnotetext{
* Corresponding author: 19210730022@fudan.edu.cn
} 
the body should not be confined to the universal standards of biomedicine, as in daily life, the body is not an individual entity simply constrained by skin and organs, but an active and complex center inside and outside the body. At the same time, the influence of cultural habits can shape the body expression. In Lock's research on menopausal aging, compared with European and American women, Japanese women in the same period are more likely to suffer from depression, which is related to the latter's more expressive social culture, while the former carries the moral of social women's need to "work hard". Thus Japanese women may face more family pressure, and will not easily express their pains. And, to a certain extent, it is the embodiment of social suffering, and also changes the biological representation, shaping people's physical signs and their judgmental interpretation. The resources needed by the physiological body are influenced by the social factors of time and space, and this process will also shape new self and new body representation. It can be said that each illness narrative brings different enlightenment.

\section{How asthma becomes parts of oneself}

In the current medical discourse, we always call for "rational, calm and educated patients". However, we have neglected that they have reestablished a more acceptable discourse system to get insight into life. To people who are suffering from illness, science, order and rational wisdom are no longer play essential roles [6].

Based on the life course and daily experience of a patient with chronic disease (asthma), this part is inspired by S. Lochlann Jain[7], the writers attempt to reveal the social and cultural characteristics of chronic diseases and how diseases enter and become a part of patients'life, and elaborate from three aspects: the social environment of illness, the social and cultural significance of illness expression, and the process of interaction between patients and asthma.

\subsection{Chronic illness and social environment}

There are social and temporal causes of chronic diseases. And its formation mechanism is rooted in personal experience and family social experience. Illness is not a simple personal experience. Instead, it is an interaction and communication, and it carries a profound social nature. The study on the significance of illness cannot be limited to personal experience, but it should be more about the social network, social situation and different ways of social life [5]. On the one hand, disease, rooted in culture, habits and the associated family relationships, is a cumulative result. On the other hand, complaint of illness has become a part of communication. Therefore, the surface meaning of symptoms is deeply rooted in the social significance and interpersonal relationship of patients'daily life, including reshaping the interaction of patients themselves.

Ms. Li, born in 1969, 51 years old, has been a civil servant in a county-level city. She has been diagnosed with asthma and mild gallbladder polyps since she was
35 years old, and her family has no genetic history. As for how to make a diagnosis and the symptoms of the disease, Ms. Li said that before she was 35 years old, if she had a cold, she would never resort to disease treatment. She had been "enduring" (procrastinating) and rarely took medicine only if she was dizzy or had a serious cough, so her condition was delayed. Her annual physical examination of the institution covering some routine items showed no abnormal symptom, so she paid no attention to chronic disease or did not need to be vigilant to other potential disease. Until she once had a "cold" for a long time, which lasted for about 2 months without any improvement, Ms. Li began to realize something unusual.

After diagnosis, the doctor prescribed some drugs for rhinitis, and told Ms. Li to keep warm at ordinary times, not to inhale cold air, and to see a doctor in time if she is sick. Ms. Li said that there is no radical medicine or other methods for her chronic asthma. The disease does not attack regularly, but the symptoms are basically the same.

Two kinds of conditions usually lead to the attack: one is that when the weather is cold, I accidentally inhale cold air; the other one happens when I walk in a hurry. This means that I can't breathe. If so, I will cough for an hour or two, and I will have a runny nose, and I can't do anything. At this time, I fail to feel myself. I am especially afraid of cold with profuse sweating. There will also be a sudden lack of breathing and face bronchial blockages. This is very dangerous. If people don't breathe, they may not be able to live.

Since the "stimulating mechanism" of asthma attack is related to the smoothness of breathing, temperature and surrounding wind speed, Ms. Li is easy to suffer it in the evening, which is also the reason why she seldom goes out at this time. Once she is stricken by the attack, she can only "obediently" stay in bed without using any cold water, and needs to constantly replace the back of the sweat towel, so a stack of toilet paper will be used up just in one or two nights. The experience during the attack is undoubtedly a kind of torture for Ms. Li. Every attack will also deepen her worry about the consequences of asthma. As she said, asthma is very dangerous. She can only breathe with her mouth, threatening her respiratory system and life.

To some extent, Ms. Li's asthma is the cumulative consequence of her long time non-treatment of cold, which is not because of her "laziness", but is that she has no time for caring herself. Ms. Li has a daughter, and before university, she gave her daughter meticulous concern. Meanwhile, she has to care for the health of her parents, husband, and husband's family members. She has always taken the role of energy output, so she receives less care from the family. In addition, she has no time to take care of her body due to her busy job. Ms. Li said she has always felt that other people's affairs are more important than her own, and she is ill when she is about to take care of herself.

Ms. Li's gallbladder also has some problems, in the physical examination organized by her institution, once the doctor found out multiple polyps in her body. The doctor suggested that the gallbladder be removed, but 
Ms. Li was very afraid of surgery and pain. Even going to the hospital for an injection would make her panic. Although the colic caused by the gallbladder pain was enough to make her unable to resist, compared with the resection operation, and the gallbladder pain did not attack much, she ignored it. What she can do in her daily life is to control her diet and avoid high-fat foods, especially those with egg yolks. As for the problem of gallbladder, she felt that it was produced about by her "unhealthy" lifestyle when she was young.

Eating too much fat can explain Ms. Li's gallbladder pain, and the reason why she likes eating fat is that she suffered too much from starvation when she was a child. Ms. Li, who was born in the late 1960s, mentioned that in the era of "material shortage", she could only eat at most a few drops of soy sauce mixed with cold rice, then carry it to school for lunch. So when she grew up, she wanted to "eat" what she did not eat enough.

From the development of asthma of Ms. Li, in the situation of chronic diseases, illness is always embodied in specific life course, and is inseparable from the specific living environment and her previous life style. The formation of her lifestyle indicates family norms and expectations of relatives. She is willing to devote herself and finally give rise to her current poor physical state. And her experience is narrated and recorded. She is recalling, and she also gives meaning to her personal experience in her own way, so as to understand herself and construct new identity and life scene, serving as a kind of subjective repair.

\subsection{Somatization: life pain and salvation}

The specific features of chronic diseases, such as time, degree and pain, vary from person to person and are embedded in people's life. The concept of somatization, proposed by Arthur Kleinman, refers to the connection between social and physiological experience: one situation, patients complain about their physical illness, but lack any pathological basis-whether it's conscious behavior (for example, pretending to be sick to avoid work, which is infrequent and easy to be exposed), or unconscious expression of troubles in life (the so-called transformation syndrome); the other situation, patients who have experienced physiological abnormalities caused by medical or mental diseases exaggerate their symptoms and their adverse consequences to an unexplained level. This exaggeration is often unconscious[8]. In other words, the writer interprets the pain of the disease as the alarm for the patients, and externalize into the expression of symptoms and "diagnosis". In addition, when the disease becomes a part of the body, it is the expression and somatization of the patients'life pain and social relationship. Moreover, the experience of illness is the real experience of different individuals, which varies from person to person.

Ms. Li has a younger brother, and her parents give more preference to him. Naturally, Ms. Li has taken on the task of doing the housework and taking care of her brother for early time. As a small kid, when her parents went out to earn work points in the field, she would go to pick pigweed, steal pears and collect coals with a basket on her back. She said that her childhood dream was to pick pigweed in a cool and comfortable place in summer.

For Ms. $\mathrm{Li}$, it is quite valuable to have the current work and family, the things she also wants to protect. So whether it's tedious housework, family conflicts or meets obstacle created deliberately by others at work, she sometimes cares, and sometimes she will tolerate it. "One step back for boundless sky" is her life principle and the primary concept in educating her children. Ms. Li said she is the representative of late marriage and late childbearing. She and her ex-husband are college classmates and gave birth to a daughter when she was nearly 28 years old. In daily life, her husband not only does not help with housework, but also goes out early and comes back late, "relying on the higher salary of tobacco company", he makes a god of his belly, is involved in gambling and playing mahjong.

Furthermore, Ms. Li's mother-in-law also has the traditional preference for boys and looks down on her and her daughter.Therefore, Ms. Li's married life is not satisfactory, and she usually does not have much time to care for her health. In the past, there was no hot water for washing up, and the tap was on the roof, causing the problem of her cold body. All the year round, her hands and feet are cold. Now in winter, four or five pieces of warm material have to be pasted in the room with hot air conditioning. The family is unreliable. Ms. Li gradually puts all her efforts on her daughter and wants to save more money for her daughter. After work, she would do some part-time jobs, such as opening a beauty salon with others. But after her mother-in-law and sister-in-law knew about it, they began to make trouble in the beauty salon, hoping to get a share through it. The good prospect did not last long. Ms. Li was ashamed and afraid that the job would be exposed and affect the work of civil servants, so she sold the beauty salon.

Some psychological and social factors often play decisive roles in the deterioration of the illness. These psychological factors include helplessness, impatience and abandonment; social factors include serious threat to survival, damage to social support and depressed social relations, which contribute to the vicious circle of psychological and physiological imbalance [8]. Ms. Li's asthma became serious when her daughter went to high school. Her daughter, with her dream, has had excellent academic performance since childhood. She was enrolled by the leading science class of a famous high school in the provincial capital, which made Ms. Li feel proud, and her economic burden and life pressure increased.

Misfortunes never come single. At this time, Ms. Li's father has suffered from lung cancer, and had an operation in Changsha. The expensive surgery and the nursing brought heavy burden to Ms. Li. In 2012, Ms. Li's physical and mental endurance declined sharply due to her accompanying study, nursing and the worry and pain of losing her father at any time. Every week, she traveled back and forth between the two cities, and her weight dropped from $46 \mathrm{~kg}$ to $40 \mathrm{~kg}$, the woman, 158 in height, was reduced almost to skeleton. In order to make 
everything smooth at home, she had to work from dawn to night, so she chose to endure minor illness and pain.

But one day, Ms. Li found it more and more difficult to recover from her illness. She felt that she was old, her immunity had declined, and she was no longer able to resist cold and hunger as she was when she was young. The endless asthma and cough attack led to the fact that she only could breathe with her mouth, which is easy to inhale dust. She was extremely uncomfortable, and felt that she completely burnt herself out.

The feeling of coughing out of breath is like the heavy pressure of life.

Although Ms. Li can tolerate many things, she is a naturally competitive person, serving as the reason why family norms and social expectations always play a role in her body and concepts. When I was young, I seldom felt sick. Even though I was tired, I still had the strength to rush forward. She has a strong sense of responsibility. As far as she is concerned, the "unimportant matters" at home is her "business". Only when the family is well, can she be well. In the past, although she failed to solve the quarrels between her mother-in-law and her husband's sister, and her father and mother, she always tried to deal with it. It seems that people around her will talk to all the things they have met, and she would keep in mind what others say. What she worried about all the time is not only work but also family relations, especially her daughter's daily life and study. Over time, the healing and maintenance of her body had also been delayed from the effects of her fatigueless.

Rather than being considered omnipotent, she felt that she should live as a Superman. Although she is in charge of her family and always gives her enthusiasm and ideas to others, her family is often ungrateful-they take these for granted, and often blame her if she do anything wrong. Ms. Li's ex-husband is a real "male chauvinist pig". If she disagrees with his work, their discussion will turn into a quarrel. If his work got better, he seldom appeared at home. I could not manage my mother's family affairs. Too much attention would cause offence from my younger brother. He always fantasizes that Ms. Li is competing with him for property.

For Ms. Li, her father's death means the loss of her spiritual support. Because she was too sad, one eye was injured because of her crying - the blood vessel in her left eye was broken, and she had an operation. The opening range was half smaller than that of her right eye, and she never wore eye makeup from then. The previous bravado, frustration and pressure were released after her father left. She left her ex-husband with a clear conscience.

It seems that the transition from illness to inhibition (refers to an internal health improvement system, which has not received enough research and attention) is often associated with the enhancement of social support, selfefficacy and renewed hope. In the remission stage, Ms. Li's anxiety and depression were also alleviated. Her sense of self-control has been enhanced, and the myth of curing illness has been replaced by the practical medical concept of nursing and reducing disability.

Ms. Li later married Mr. Shen, who offered much money, contacts and emotional support during her father's treatment. His gentleness has given Ms. Li a lot of comfort and protection. The slow pace of life gives her the right to get sick "without any scruples", and she feels that her body and mind have been redeemed.

Therefore, the author thinks that the distinction between "disease" and "illness" is the key, and an essential difference is that diseases and other physical abnormalities ignore the relevant social and psychological factors. While illness is a deeper individual experience, which refers to the fresh experience and the patient's personal feelings: terrible symptoms, fear, family distress and economic pressure. Arthur Kleinman believes that the first thing patients experience when they are sick is "illness". And such "illness" will be affected by social concepts, cultural norms and personal character when patients communicate with family members and friends, so it is a concrete and self-directed personal experience [8].

What the patient experienced is illness, which is not confirmed by the doctor, and experienced by the individual with subjective feature. The first level of symptoms implies the knowledge of the body and self, as well as the relationship between them and our intimate life. Meanwhile, physical experience has also become the embodiment of social culture. Through specific values, social control is internalized, political form is materialized, becoming the physical feeling and physiological needs. Therefore, illness is not only manifested in the body pain, but also carries social significance. Under the pressure of life, Ms. Li's emotional tolerance has become the intolerance and nonattack of disease. However, the new round of asthma attack is not a bad thing for her to some extent thanks for the new marriage, her spiritual remedy. As her husband serves as the solid foundation for her life, she is willing to and starts to accept treatment. She can also put the resistance to her surroundings behind and begins to enjoy life, of course, begins to cherish life.

\subsection{The transformation of healing methods and the construction of view on disease}

Arthur Kleinman focuses on the narration of illness with the purpose of exploring the patients'life experience, so as to point out the more important social and cultural significance related to disease. Therefore, he does not emphasize the treatment process and the story behind it. In this part, the author introduces the patient's way of healing and the choppy experiences, because the choice of the patient's healing method is closely related to her social network, and with the change of the method, the patient's view of health and disease will also be reflected and shaped.

Ms. Li first went to the hospital for treatment when she felt uncomfortable. In the course of treatment, her condition also improved. As a patient with chronic physical symptoms, Ms. Li, after undergoing modern medical scientific diagnosis and treatment, has to constantly explore her own personal healing methods in folk prescriptions, from miracle-working doctor and 
traditional Chinese medicine nursing. So, what happened to Ms. $\mathrm{Li}$ in the process? What is her course of mind?

Ms. Li's first choice of treatment is modern medical treatment. She thinks it is more standardized and can use the medical insurance card. But she only goes there when she needs first aid. The reason is that the type of medicine prescribed and the diagnosis are single, and the treatment fails to cure the root cause. Sometimes it is impossible to identify whether the cough is cured automatically or by the medicine, so I do not put all my hope on the treatment. She has used Budesonide and Formoterol Fumarate Powder for Inhalation for nearly six years (six tubes a year). Each one cost 398 yuan a few years ago, and the price has decreased to 298 yuan thanks for the medical reform in the past two years. Ms. Li said it does not need to be used when the weather is warm, but it consumes faster in the face of dropping temperature or changing seasons. She has to use a whole tube a month under serious condition, and one tube for two months if it is not serious. Ms. Li's asthma was the most serious in 2018, she said that it developed into pneumonia and she was hospitalized.

After the twists and turns, Ms. Li felt that the disease should be treated seriously, and she could not bear any disease. She found that medical treatment was useful and decided to accept systematic treatment. She began to seek medical advice everywhere, like resorting to acquaintances, websites, various official accounts and forums. she would take any medicine as long as someone said it was useful, and was willing to try any miracle medicine.

After trying various treatment modes, Ms. Li knows it is difficult to treat the root cause, she begins to reflect on her lifestyle and turn to the daily physical nursing. She mentioned that she had never dried her hair before and only slept directly. When she lived in the countryside, there is no hair dryer or the habit of blowing-dry hair, and that she was drying it all by natural wind, all these lead to her physical weakness. She felt that she had little knowledge about health preservation. With the promotion of her friends, she tried many lifestyles that can help her breathe, such as practicing Tai Chi, doing light yoga, drinking health tea, and eating fruits every day. Besides, the awareness of caring for the body is penetrated into her daily life.

I now find that paying attention to the body at ordinary times is better to our physical health, while ignoring it must give rise to poor condition. Our body needs to rest, my previous busy life is carried out at the cost of my health and breathing.

Ms. Li's nursing way also bring her some benefits.

I used to cough when the wind blows, and gasp for breath when I climb a little higher or a mountain. But now I feel good even after climbing the sixth floor because of my improved vital capacity. I was scared and nervous when I got sick at the beginning, and only could see the doctor in the face of disease outbreak, putting me in a passive place, while now I can learn to defeat it. Exercises will help you feel the changes in life.

In addition, Ms. Li also said that good health not only makes herself feel comfortable, but is good for the whole family and her daughter.This concept makes Ms. Li more determined about the importance of taking good care of herself. Whether it is to sacrifice her time and health, or to get well, it is a contribution to the family to some extent.

It may be that asthma has both the characteristics of chronic course and acute attack, and carries strong physical, psychological and social adaptive changes. Ms. Li's experience prompts her to examine the disease based on the dual roles and understanding of doctors and patients, that is, to examine the body as well as the means of treatment. Therefore, it is of great significance to explore the process of the transformation of Ms. Li's healing methods.

\section{Conclusion}

To sum up, disease explanation is a kind of narrative reconstruction. By connecting different aspects of life, patients can repair the fracture among body, self and the world. The narration of patients is to endow personal experience with significance in their own way, so as to understand themselves and construct a new identity and life scene. Only in this way can they adapt to the new role of "patients" and find support for their future life.

\section{References}

1. Freidson, E.Profession of Medicine. New York: Dodd, Mead. (1970)

2. Zola, Irving. Medicine as an Institution of Social Control. The Sociological Review 20 (4):487504.(1972)

3. Bao Lei.Medicalization of modern daily life. Forward Position (11): 125-127. (2010)

4. $\mathrm{Xu}$ Ganlin. Expert knowledge and medical market $[\mathrm{G}] / /$ Yang Peichang,edit. Capitalism and contemporary medical treatment. Taipei: DITM Publishing House. (2009)

5. Arthur Kleinman. The Illness Narratives: Suffering, healing and human condition. Shanghai Translation Publishing House.(2018)

6. Wang Jianxin, Zhao Xuan. Discourse strategies and personality construction of the illness descriptions: a perspective of medical anthropological on patient needs. Journal of Northwest Normal University (Social Sciences), Volume 53 No. 4.(2016)

7. S. Lochlann Jain.Malignant: How Cancer Becomes Us by Berkeley: University of California Press.(2013)

8. Arthur Kleinman.Social Origins of Distress and Disease Neurasthenia, Depression and Pain in Modern China. New Haven Yale University Press.(1986) 